\title{
A Serious Mediastinum Abscess Induced by Endobronchial Ultrasound-guided Transbronchial Needle Aspiration (EBUS-TBNA): A Case Report and Review of the Literature
}

\author{
Hiroshi Ishimoto ${ }^{1}$, Kazuhiro Yatera ${ }^{1}$, Keigo Uchimura ${ }^{1}$, Keishi Oda ${ }^{1}$, Masaru Takenaka ${ }^{2}$, \\ Toshinori Kawanami ${ }^{1}$, Fumihiro Tanaka ${ }^{2}$ and Hiroshi Mukae ${ }^{1}$
}

\begin{abstract}
A 75-year-old man with interstitial pneumonia and enlarged mediastinal lymph nodes underwent endobronchial ultrasound-guided transbronchial needle aspiration (EBUS-TBNA). He developed a high-grade fever seven days after EBUS-TBNA was performed; laboratory and radiologic findings showed intense inflammatory reactions, with swelling of the mediastinal lymph nodes on chest computed tomography. Mediastinal lymph node abscess was diagnosed, and it worsened in spite of systemic antibacterial treatment. Surgical treatment using a median sternotomy was performed, and the cultivation of surgically obtained mediastinal lymph node abscess fluid revealed Streptococcus intermedius. Combined treatment with antibiotics and surgical treatment was effective, leading to remission.
\end{abstract}

Key words: endobronchial ultrasound-guided transbronchial needle aspiration (EBUS-TBNA), mediastinal lymphatic abscess, Streptococcus intermedius

(Intern Med 54: 2647-2650, 2015)

(DOI: 10.2169/internalmedicine.54.4465)

\section{Introduction}

This case report describes a serious mediastinum lymphatic abscess caused by endobronchial ultrasound-guided transbronchial needle aspiration (EBUS-TBNA).

\section{Case Report}

A 75-year-old Japanese man had experienced dyspnea during exertion for approximately six months. He had uncontrolled diabetes mellitus [HbA1c, National Glycohemoglobin Standardization Program $(\mathrm{NGSP})=9.1 \%$ ], and had a history of hepatic abscess. His vital signs were normal on admission. Bilateral fine crackles were heard with auscultation of the chest. Chest X-rays revealed a reticular shadow around both sides of the lower lung field (Fig. 1a). Chest computed tomography (CT) showed honeycomb lung forma- tions in the dorsal side of the bilateral lower lobes as well as traction bronchiectasis (Fig. 1b). Slight mediastinal lymph node hyperplasia was observed (Fig. 1c). Although his serum angiotensin converting enzyme levels were within normal limits, slightly high serum calcium levels indicated sarcoidosis as a possible cause of interstitial pneumonia; therefore, we performed EBUS-TBNA in order to carry out a pathological examination of the mediastinal lymph nodes.

Under mild sedation with midazolam, EBUS-TBNA was performed using the UC260FW (Olympus, Tokyo, Japan). The blood flow within the mediastinum lymph node was confirmed using color Doppler. A 22-G needle was used for the puncture. The puncture was made using "the style of stylet push out" method. Puncturing was performed twice in each right tracheobronchial lymph node (\#4R) and subcarinal lymph node (\#7). This was followed by bronchoalveolar lavage (BAL) in the right $S^{5}$ and trans-bronchial lung biopsies (TBLB) in the right $S^{9}$ using the 1T260 (Olympus).

${ }^{1}$ Department of Respiratory Medicine, University of Occupational and Environmental Health, Japan and ${ }^{2}$ Second Department of Surgery, University of Occupational and Environmental Health, Japan

Received for publication November 7, 2014; Accepted for publication February 22, 2015

Correspondence to Dr. Hiroshi Ishimoto, h-ishimoto@med.uoeh-u.ac.jp 

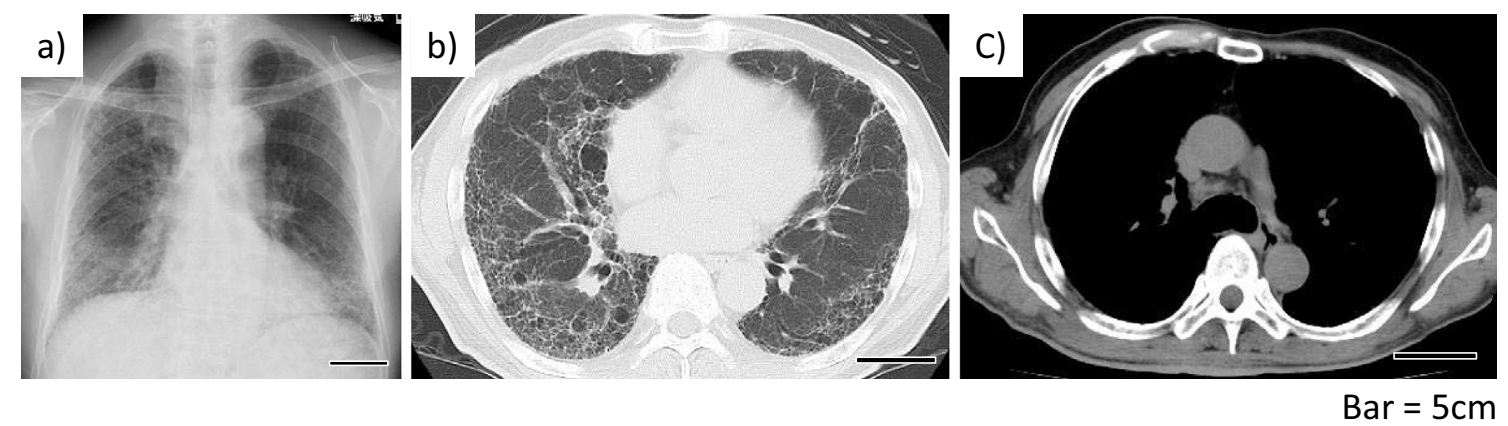

Figure 1. Chest X-ray (a) and computed tomography (b, c) images obtained at the first admission. Chest X-rays and computed tomography revealed a reticular shadow around both sides of the lower lung field (a, b). Slight mediastinal lymph node hyperplasia was observed at the \#4R lymph node (c).
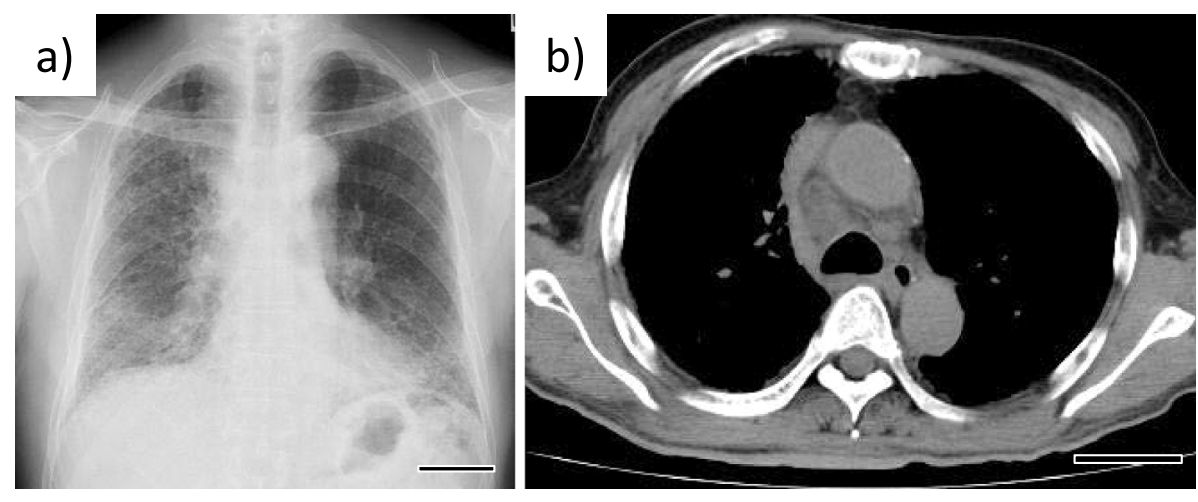

$$
\text { Bar }=5 \mathrm{~cm}
$$

Figure 2. Chest X-ray (a) and computed tomography (b) images obtained at the second admission. Superior mediastinum expansion was recognized (a). The lymph node of \#4R exhibited swelling, and a low-density area was recognized inside this lymph node (b).

Prophylactic antibiotics were not prescribed and the patient was discharged from the hospital two days after the diagnostic examination. Pathological examination of both the TBNA and TBLB specimens showed nonspecific inflammatory changes and no malignant or granulomatous findings.

The patient developed a persistent high fever seven days after the diagnostic examination, and he returned to our hospital nine days after the diagnostic examination. An advanced inflammatory reaction (white blood cell count= $18,100 / \mu \mathrm{L} ; \mathrm{CRP}=18.51 \mathrm{mg} / \mathrm{dL}$ ) was observed, and expansion of the superior mediastinum was revealed by chest Xray (Fig. 2a). Therefore, he was re-admitted to the hospital. Chest CT revealed swelling of lymph node \#4R, with a lowdensity area inside the lymph node. This indicated that mediastinum lymphadenitis had occurred, with a portion becoming abscessed (Fig. 2b). Although meropenem (3 g/day) and linezolid (1,200 mg/day) were initiated, the high fever remained along with an irregularity of the circulatory movement. Therefore, we decided to perform mediastinum lymph node dissection the third day after admission. An acute exacerbation of chronic respiratory failure in our patient did not permit one-lung ventilation during surgery. Furthermore, en bloc removal of the infected and swollen lymph nodes was important; therefore, median sternotomy was performed under general anesthesia. Following release of the superior vena cava and the ascending aorta, swelling of the \#4 lymph node was exposed. The lymph node was punctured aseptically, and the pus inside the lymph node was collected. The \#4 lymph node and the paratrachial lymph node (\#2) were removed en block. The inflammation had spread to some of the fat tissues surrounding the lymph nodes.

A culture of the pus from the mediastinal lymph node yielded growth of Streptococcus intermedius. Therefore, cefazolin (4.5 g/day) and clindamycin (1,200 mg/day) were administered for two weeks. Thereafter, doripenem ( $3 \mathrm{~g} /$ day) was administered for two weeks for concomitant aspiration pneumonia.

\section{Discussion}

Compared with mediastinoscopy, EBUS-TBNA is a minimally invasive method for the investigation of mediastinum lymph node swelling and thus has recently become increasingly more popular. Furthermore, EBUS-TBNA characteristically has few complications. Only a few reports have described infectious complications of the mediastinum (1-6) 
Table. The Case List of the Infectious Complication Caused by Endbronchial Ultrasound Guided Transbronchial Needle Aspiration.

\begin{tabular}{|c|c|c|c|c|c|c|}
\hline age & $\operatorname{sex}$ & Lapsed days & The kind of infectios disease & Bacteria of a cause & $\begin{array}{c}\text { Surgical } \\
\text { procedure }\end{array}$ & Reference \\
\hline 50 & $\mathrm{M}$ & 19 & pericardiocentesis & $\begin{array}{l}\text { Actinomyces odontolytius } \\
\text { Streptococcus mutans }\end{array}$ & drainage & 1 \\
\hline 58 & $\mathrm{M}$ & 9 & abscess in the lung mass & N.D. & - & 1 \\
\hline 89 & $\mathrm{~F}$ & 14 & mediastinal abscess & $\begin{array}{c}\text { alpha streptococcus } \\
\text { Diphtheroids }\end{array}$ & operation & 2 \\
\hline 68 & $\mathrm{M}$ & 35 & mediastinal abscess & $\begin{array}{c}\text { Candida albicans } \\
\text { Y-hemolytic Streptococcus }\end{array}$ & - & 3 \\
\hline 66 & $\mathrm{M}$ & 5 & mediastinal abscess & $\begin{array}{c}\text { Propionibacterium acnes } \\
\text { Bacteroides } \\
\text { Eubacterium species }\end{array}$ & operation & 3 \\
\hline 68 & M & 60 & mediastinal abscess & Streptococcus viridans & operation & 4 \\
\hline 59 & $\mathrm{M}$ & 7 & mediastinal abscess & N.D. & - & 5 \\
\hline 55 & $\mathrm{~F}$ & 6 & pericardiocentesis & Streptococcus viridans & operation & 6 \\
\hline 59 & M & 10 & pericarditis and pneumonia & $\begin{array}{l}\text { Group C beta-haemolytic } \\
\text { Streptococcus species }\end{array}$ & - & 6 \\
\hline 75 & $\mathrm{M}$ & 9 & mediastinal abscess & Streptococcus intermidius & operation & Our Case \\
\hline
\end{tabular}

(Table).

Investigations to confirm bacteremia have been carried out to examine the occurrence of infectious disease due to endoscopic maneuvers utilizing this technique (7). For EBUSTBNA, a previous investigation confirmed the presence of bacteremia in a blood culture obtained during the diagnostic examination (8). The frequency of the occurrence of bacteremia does not differ greatly between EBUS-TBNA and conventional bronchoscopy. Therefore, bacterial invasion from the mucous membrane is thought to be responsible for bacteremia (9). However, these studies have limitations, and the method for verifying bacteremia is not the same as that for verifying the occurrence of mediastinum lymphadenitis due to EBUS-TBNA. Because the frequency of the occurrence of infectious complications, including mediastinum lymphadenitis, is very low $(0.19 \%)(10)$, verifying mediastinum lymphadenitis is difficult. However, mediastinum lymphadenitis should be carefully considered in the differential diagnosis because it is a serious complication with occasionally fatal outcomes (6).

The bronchoscope is placed into the trachea through the upper airway; therefore, maintaining aseptic conditions during this procedure is difficult. Moreover, because an ultrasonic endoscope is a heteroscope, insertion is difficult in comparison with usual bronchoscopy. One factor that influences contamination of the tip of the fiberscope is the approach after the tracheal intubation. However, past research has shown that the occurrence of bacteremia due to tracheal intubation is beyond $10 \%$ (10). Furthermore, deep anesthesia is unnecessary when a diagnostic examination is performed with EBUS-TBNA. The absorption of any saliva or fluid in the intraoral region before bronchoscope insertion is a fundamental maneuver, and the elevation of the lower jaw of the patient by the assistant is important for this step. After encountering serious complications associated with stylet handling, we discontinued the use of "the style of stylet push out" and assigned stylet handling to an independent assistant wearing sterile gloves to prevent contamination of the mediastinal lymph nodes with the needle and stylet at our hospital.

The existence of uncontrolled diabetes mellitus would influence this critical illness. Antibacterial medication is recommended as a prophylaxis for infectious endocarditis, corresponding to the patient's risk factors (11). For the bronchoscope, a previous investigation showed no validity for using an antibacterial agent for prophylactic medication (12). However, a satisfactory evaluation cannot presently be carried out for EBUS-TBNA. In high-risk patients, such as our patient who had uncontrolled diabetes mellitus, we believe that prophylactic antibiotics should be administered to avoid serious complications such as mediastinitis, as observed in our patient. In addition, in recent report of pericarditis as a fatal complication of EBUS-TBNA, antibiotic prophylaxis was recommended to avoid the possible occurrence of serious acute pericarditis in patients who underwent EBUS-TBNA with necrotic lymph nodes located in the middle mediastinum (6). The intraoral resident florae become the origin bacteria in many cases (Table). Therefore, cefazolin and a ceftriaxone should be recommended according to the guidelines of the American Heart Association (7).

The authors state that they have no Conflict of Interest (COI). 


\section{References}

1. Haas AR. Infectious complications from full extension endobronchial ultrasound transbronchial needle aspiration. Eur Respir J 33: 935-938, 2009.

2. Moffatt-Bruce SD, Ross P Jr. Mediastinal abscess after endobronchial ultrasound with transbronchial needle aspiration: a case report. J Cardiothorac Surg 5: 33, 2010.

3. Kouskov OS, Almeida FA, Eapen GA, Uzbeck M, Deffebach M. Mediastinal infection after ultrasound-guided needle aspiration. J Bronchology Interv Pulmonal 17: 338-341, 2010.

4. Huang CT, Chen CY, Ho CC, Yu CJ. A rare constellation of empyema, lung abscess, and mediastinal abscess as a complication of endobronchial ultrasound-guided transbronchial needle aspiration. Eur J Cardiothorac Surg 40: 264-265, 2011.

5. Gochi F, Chen F, Aoyama A, Date H. Mediastinal infectious complication after endobronchial ultrasound-guided transbronchial needle aspiration. Interact Cardiovasc Thorac Surg 17: 751-752, 2013.

6. Lee HY, Kim J, Jo YS, Park YS. Bacterial pericarditis as a fatal complication after endobronchial ultrasound-guided transbronchial needle aspiration. Eur J Cardiothorac Surg. (in press).

7. Yigla M, Oren I, Bentur L, et al. Incidence of bacteraemia follow- ing fibreoptic bronchoscopy. Eur Respir J 14: 789-791, 1999.

8. Steinfort DP, Johnson DF, Irving LB. Incidence of bacteraemia following endobronchial ultrasound-guided transbronchial needle aspiration. Eur Respir J 36: 28-32, 2010.

9. Asano F, Aoe M, Ohsaki Y, et al. Complications associated with endobronchial ultrasound-guided transbronchial needle aspiration: a nationwide survey by the Japan Society for Respiratory Endoscopy. Respir Res 14: 50, 2013.

10. Valdes C, Tomas I, Alvarez M, Limeres J, Medina J, Diz P. The incidence of bacteraemia associated with tracheal intubation. Anaesthesia 63: 588-592, 2008.

11. Wilson W, Taubert KA, Gewitz M, et al. Prevention of infective endocarditis: guidelines from the American Heart Association: a guideline from the American Heart Association Rheumatic Fever, Endocarditis, and Kawasaki Disease Committee, Council on Cardiovascular Disease in the Young, and the Council on Clinical Cardiology, Council on Cardiovascular Surgery and Anesthesia, and the Quality of Care and Outcomes Research Interdisciplinary Working Group. Circulation 116: 1736-1754, 2007.

12. Park JS, Lee CH, Yim JJ, et al. Impact of antibiotic prophylaxis on postbronchoscopy fever: a randomised controlled study. Int J Tuberc Lung Dis 15: 528-535, 2011.

(C) 2015 The Japanese Society of Internal Medicine http://www.naika.or.jp/imonline/index.html 